\title{
Correlation Between Level of Interleukin-37 and Rheumatoid Arthritis Progression
}

\author{
Jifeng Zhu \\ Chuanjiang Xie \\ Hongjiu Qiu \\ Ling Shi
}

Orthopedic Surgery, Daping Hospital, Army Medical University, Chongqing, 400042, People's Republic of China
Correspondence: Ling Shi

Orthopedic Surgery, Daping Hospital, Army Medical University, Chongqing, 400042, People's Republic of China

Tel +86 23-68729330

Email lingshi.amu@outlook.com
Background: Rheumatoid arthritis (RA) is a systemic autoimmune inflammatory disease that primarily affects joints. Interleukin-37 (IL-37) is an anti-inflammatory cytokine that is known to suppress immune response and inflammation. The objective of this study is to evaluate the correlation between level of IL-37 and RA progression using the disease activity score in 28 joints (DAS-28).

Methods: A total of 87 RA patients were separated into 4 groups based on the DAS28, referred to as the remission, mild, moderate and severe groups. 18 healthy volunteers were also included. Serum level of IL-37 and IL-37 mRNA expression level in peripheral blood mononuclear cells (PBMCs) in each individual participant as well as IL-37 mRNA expression level in synovial cells were assessed to explore their correlation with RA progression.

Results: Serum level of IL-37 and IL-37 mRNA expression levels in both PBMCs and synovial cells were all positively correlated with the severity of RA as reflected by the DAS28. Receiver operating characteristic (ROC) analysis revealed area under curve (AUC) values of $1,0.5262$ and 0.7789 for the three parameters.

Conclusion: Our results suggest that serum IL-37 level and mRNA expression levels of IL37 in PBMCs and synovial cells are correlated with the severity of RA in a Chinese population.

Keywords: rheumatoid arthritis, interleukin-37, DAS-28, PBMC, synovial cell

\section{Introduction}

Rheumatoid arthritis (RA) is a chronic autoimmune disease, in which the body's own immune system attacks the body's own joints. Unlike the mechanic-induced osteoarthritis that usually occurs in late life, RA can arise at any time. It is characterized by synovial cell over-proliferation and lymphocyte invasion that result in progressive damage to the articular cartilage and subsequent bone erosion. In addition to joints as primary targets, it can also cause other complications, including rheumatoid nodules, pulmonary defects, blood vessel inflammation and systemic comorbidities. ${ }^{1}$ A number of cell types are known to be involved in RA pathogenesis, including synovial fibroblasts, osteoclasts, immune-related $\mathrm{T}$ and B lymphocytes and macrophages. Orchestration of these cells stimulates the release of various inflammatory mediators that sustain the chronic inflammatory response of the disease. Cytokines, including both pro- and anti-inflammatory ones are known to play fundamental roles in RA progression via inflammation and articular cartilage destruction. ${ }^{2}$ The efficacy of biological disease-modifying antirheumatic drugs (bDMARDs) that target cytokines has been demonstrated in patients with RA. ${ }^{3}$ 
RA progression can be classified into 4 stages. Stage 1 is an early stage of RA where there is inflammation in the synovial membrane, but no damage to cartilage or bone is detected yet. Stage 2 is a moderate stage of RA where the articular cartilage is damaged by the synovial inflammation. Stage 3 is a severe stage of RA where both the articular cartilage and the underneath bones are damaged. Stage 4 is the end stage of RA where the joint inflammation disappears and the joint fails to function any longer. Reduced muscle strength, structural damage to the joint and bone fusion are often observed at this stage.

Currently, Disease Activity Score (DAS) is the major scoring system recommended by the European League Against Rheumatism (EULAR) for evaluating the disease activity of RA. DAS28 is a modified version of DAS that examines 28 joint based on the number of swollen and tender joints, visual assessment of patients' disease severity and the erythrocyte sedimentation rate (ESR). ${ }^{4}$ ESR is employed to reflect the degree of inflammation. The 4 stages of RA can be distinguished based on the DAS28 score: DAS28<2.6 in stage $1, \mathrm{DAS} 28 \leq 3.2$ in stage 2, DAS $\leq 5.1$ in stage 3 and DAS28 $>5.1$ in stage 4 .

Interleukin-37 (IL-37), a member of the antiinflammatory cytokines, is initially produced as a precursor protein that is later processed by Caspase-1 to release its mature form. ${ }^{5}$ Functions of IL-37 have been implicated in a number of tissues and immune cell types, such as bone marrow, thymus, lymph nodes, monocytes and B lymphocytes. ${ }^{6}$ Its role in suppressing the production of various cytokines involved in inflammatory response has been proposed in different in vivo and in vitro models. ${ }^{7-11}$ Elevated serum IL-37 level has been shown to be closely related to inflammatory cytokines and RA progression. ${ }^{12}$ Upon anti-RA drug treatment, serum IL-37 level could be greatly reduced in RA patients. ${ }^{13}$ However, how levels of IL-37 correlate with the severity of RA remains unclear. In the present study, we aim to investigate the correlation between the IL-37 levels in serum and synovial tissues and the DAS28 scores in RA patients with different severities.

\section{Methods}

\section{Patients and Study Design}

The study was approved by the ethical committee of Daping hospital (2018-97254) and performed in accordance with the Chinese regulation and Helsinki declaration. Verbal consent was obtained from all patients. The purpose and design of the study was carefully explained to all participants accompanied by their close relatives before verbal consent was obtained according to the guidance provided by the ethical committee. Verbal consent was selected due to the limited writing ability of some participants. This procedure has been approved by the ethical committee.

A total of 87 RA patients recruited at our hospital and 18 healthy volunteers were included in the study. DAS28 was assessed for all RA patients, based on which they were divided into 4 groups: remission (DAS28 $\leq 2.6)$ mild $(2.6<\mathrm{DAS} 28 \leq 3.2), \quad$ moderate $(3.2<$ DAS $28 \leq 5.1)$ and severe (DAS28 $>5.1)$. Blood samples were collected from all participants. Peripheral blood mononuclear cells (PBMCs) were collected from all participants by Ficoll-Paque premium (Sigma) and density gradient centrifugation under sterile conditions. Synovial tissues were collected from RA patients in the severe group who suffered from erosive inflammation and underwent surgery. Synovial cells were immediately isolated from the synovial tissues as previously described $^{14}$ for RNA extraction. Briefly, after surgery pieces of the superficial layer of the synovium were collected for collagenase digestion. The digested tissue was filtered through a $0.2 \mu \mathrm{m}$ filter to collect the synovial cells.

\section{Serum IL-37 Assessment}

Blood samples were stabilized and fractionated into serum and blood cells by centrifugation. Serum was collected and stored at $-80^{\circ} \mathrm{C}$ until use. IL-37 concentration was detected by enzyme-linked immunosorbent assay (ELISA) kits according to the manufacturer's instructions (AdipoGen, Liestal, Switzerland). Briefly, microtiter plates were coated with horse radish peroxidase (HRP)conjugated antibody against IL-37 before samples were added and incubated for 2 hours at $37^{\circ} \mathrm{C}$. TMB substrate was subsequently added for the development of color reaction. OD values at $450 \mathrm{~nm}$ were recorded.

\section{RNA Extraction and RT-PCR}

Total RNA extraction from PBMCs and synovial cells, cDNA synthesis and RT-PCR for IL-37 level were performed as previously described. ${ }^{15}$ The $2^{-\Delta \Delta \mathrm{Ct}}$ method was used to determine the relative expression of IL-37. PCR products were verified by melting curve analysis. The relative expression of IL-37 was normalized to $\beta$-actin. The following primer sequences were used: 
IL-37:

5'-AGTGCTGCTTAGAAGACCCGG-3'; 5'-AGAGTC CAGGACCAGTACTTTGTGA-3'

$\beta$-actin:

5'-CCTGACTGACTACCTCATGAAG-3'; 5'-GACGT AGCACAGCTTCTCCTTA-3

\section{Statistical Analysis}

GraphPad Prism 7 was used to analyze all the data. One-way ANOVA combined with post hoc test, correlation analysis and receiver operating characteristic (ROC) were performed. D'Agostino's K-squared test was used to confirm the normal distribution of the data. Levene's test was performed to confirm the homogeneity of variance for all samples. A P value less than 0.05 was considered statistically significant.

\section{Results}

\section{General Features of Participants}

Characteristics of all participants, including RA patients in the 4 groups and the healthy volunteers, were listed in Table 1. In general, age, gender and body mass index (BMI) were all comparable among the participants of the 5 groups, with a $P$ value of $0.9934,0.8562,0.3122$, respectively.

\section{Correlation Between Serum IL-37 Level and DAS28}

We first compared the IL-37 level in the serum among the participants in the 5 groups and found that the more severe the RA progression in the patients the higher the IL-37 level was present in their blood, where the healthy volunteers had the lowest (Figure 1A). One-way ANOVA analysis revealed a $P$ value less than 0.0001 for the comparison among the 5 groups. Then, we performed correlation analysis between the serum IL-37 level and the DAS28 in all RA patients and revealed a positive correlation between the two factors (Figure 1B). Next, we plotted the ROC to further assess the potential of serum IL-37 as a diagnostic factor for RA and revealed an area under curve (AUC) value of 1 (Figure 1C).
Taken together, these data suggest that the serum IL-37 level is tightly correlated with the severity of RA as reflected by the DAS28 and has a great potential to be used as a diagnostic factor for RA.

\section{Correlation Between IL-37 mRNA Level in PBMC and DAS28}

To further confirm the role of blood IL-37 level in RA, we assessed the mRNA level of IL-37 in the PBMCs of the participants in the 5 groups. Consistently, it also increased with the increasing RA severity and remained at the lowest level in the healthy volunteers (Figure 2A). One-way ANOVA analysis revealed a $\mathrm{P}$ value less than 0.0001 for the comparison among the 5 groups. Correlation analysis revealed a positive correlation between the IL-37 mRNA level in PBMC and the DAS28 (Figure 2B). In addition, an AUC value of 0.5262 was identified in the ROC (Figure $2 \mathrm{C})$. These data further support the expression of IL-37 in blood-related cells as a diagnostic factor for RA.

\section{Correlation Between IL-37 mRNA Level in Synovial Cell and DAS28}

Given that the synovial level of IL-37 has been proposed to be associated with osteoarthritis, ${ }^{16}$ we wondered whether it also affects the progression of RA. Therefore, we assessed the mRNA level of IL-37 in the synovial cells of the patients in the severe RA group who underwent surgical treatment and correlated it with the DAS28 in these patients. Indeed, we revealed a positive correlation between the two factors (Figure 3A) and an AUC value of 0.7789 was identified in the ROC (Figure 3B).

Taken together, these data further suggest that IL-37 expression level in the synovial cells also can be a potential candidate for RA diagnosis.

\section{Discussion}

RA is a systemic and chronic autoimmune disease characterized by inflammation, cell infiltration, excessive synovial cell

Table I Characteristics of All Participants

\begin{tabular}{|l|l|l|l|l|l|l|}
\hline Characteristics & $\begin{array}{l}\text { Remission } \\
(\mathbf{n = 2 0 )}\end{array}$ & $\begin{array}{l}\text { Mild } \\
(\mathbf{n = 2 3 )}\end{array}$ & $\begin{array}{l}\text { Moderate } \\
(\mathbf{n = 2 2})\end{array}$ & $\begin{array}{l}\text { Severe } \\
(\mathbf{n = 2 2})\end{array}$ & $\begin{array}{l}\text { Healthy Volunteers } \\
(\mathbf{n}=1 \mathbf{8})\end{array}$ & P value \\
\hline Age (year) & $50.15 \pm 11.32$ & $51.17 \pm 14.42$ & $49.36 \pm 12.4$ & $50.09 \pm 11.94$ & $50.33 \pm 12.54$ & 0.9934 \\
Gender (Male:Female) & $11: 9$ & $11: 12$ & $12: 10$ & $13: 9$ & $9: 9$ & 0.8562 \\
BMI (kg/m2) & $23.01 \pm 1.7$ & $22.74 \pm 1.75$ & $23.62 \pm 1.84$ & $22.92 \pm 1.92$ & $22.53 \pm 1.15$ & 0.3122 \\
DAS28 & $1.86 \pm 0.52$ & $2.92 \pm 0.14$ & $4.09 \pm 0.58$ & $6.87 \pm 1.07$ & & \\
\hline
\end{tabular}



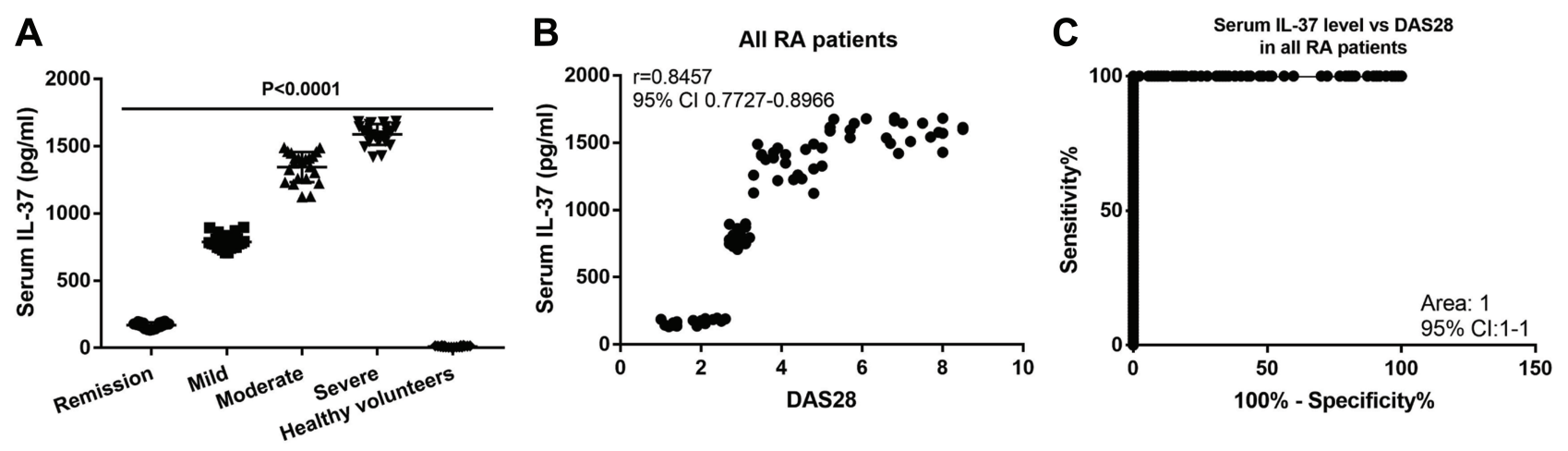

Figure I Serum IL-37 level is positively correlated with the RA progression. (A) Serum IL-37 level comparison in the 5 groups of participants. P<0.000I is the One-way ANOVA analysis among the 5 groups. (B) Correlation analysis of serum IL-37 level and the DAS28 in all RA patients. (C) ROC of serum IL-37 level and the DAS28 in all RA patients.
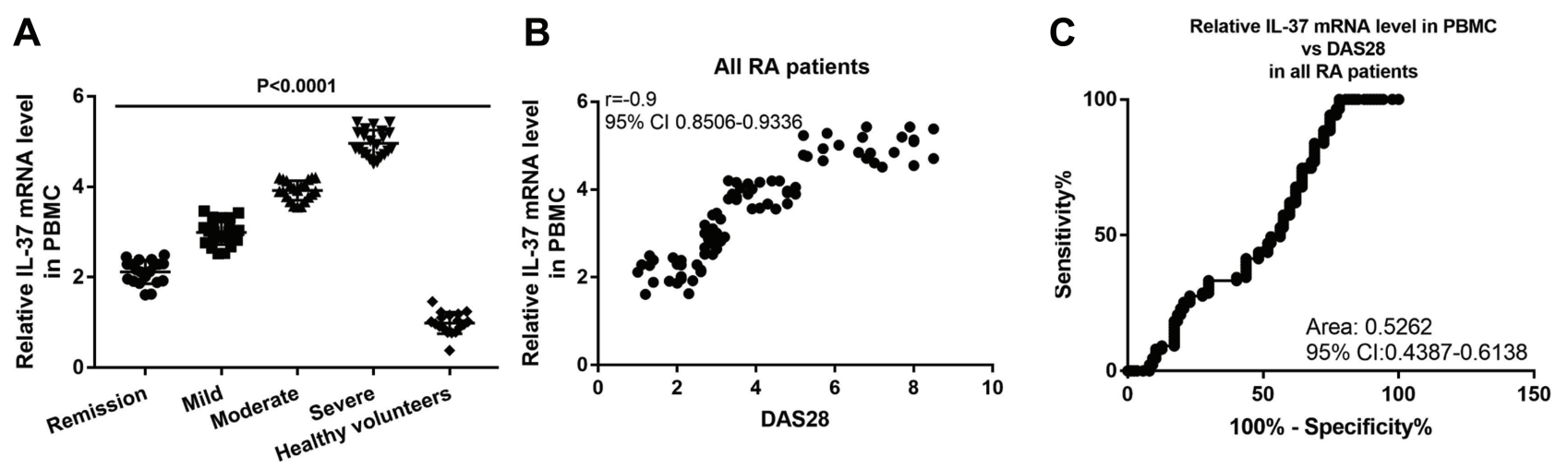

Figure 2 IL-37 mRNA expression level in PBMC is positively correlated with the RA progression. (A) IL-37 mRNA expression level in PBMC comparison in the 5 groups of participants. $\mathrm{P}<0.000 \mathrm{I}$ is the One-way ANOVA analysis among the 5 groups. (B) Correlation analysis of IL-37 mRNA expression level in PBMC and the DAS28 in all RA patients. (C) ROC of IL-37 mRNA expression level in PBMC and the DAS28 in all RA patients. Y axis in (A) and (B) is the $2^{-\Delta \Delta C t}$ value of IL-37 normalized to the $2^{-\Delta \Delta C t}$ value of $\beta$-actin.

A

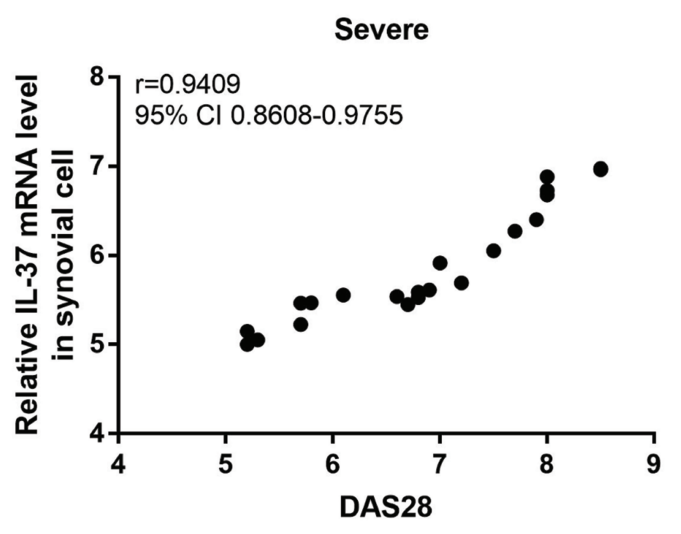

B Relative IL-37 mRNA level in synovial cell vs DAS28 in Severe RA patients

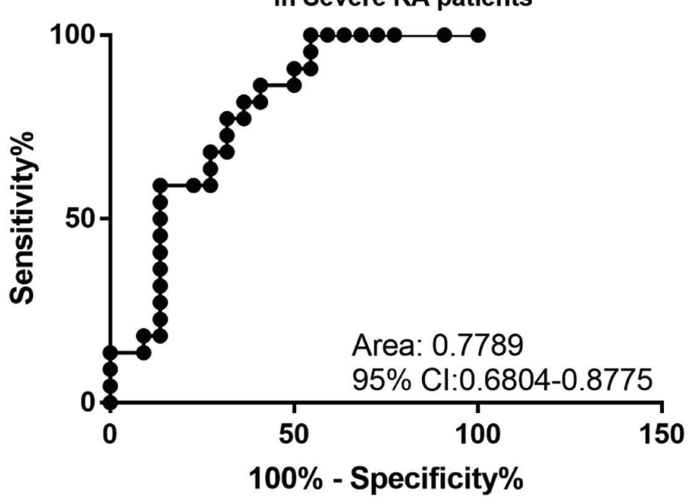

Figure 3 IL-37 mRNA expression level in synovial cells is positively correlated with the RA progression. (A) Correlation analysis of IL-37 mRNA expression level in synovial cells and the DAS28 in RA patients of the severe group. Y axis is the $2^{-\Delta \Delta C t}$ value of IL-37 normalized to the $2^{-\Delta \Delta C t}$ value of $\beta$-actin. (B) ROC of IL-37 mRNA expression level in synovial cells and the DAS28 in RA patients of the severe group.

proliferation and bone destruction. Many studies have linked the elevated levels of inflammatory cytokines with RA progression, ${ }^{17,18}$ among which IL-37 has been proposed to play an important role. ${ }^{9,13,15,19}$ However, these studies are limited by their small sample size and a clear correlation between IL-37 and RA progression in patients with differential 
RA severities. In the present study, we explored the potential of IL-37 as a diagnostic factor for RA. We found that the serum level of IL-37 and the mRNA levels of IL-37 in both PBMCs and synovial cells were all positively correlated with RA severity as reflected by the DAS28. Results of ROC analysis strongly support the IL-37 levels in all three tested compartments are potential diagnostic factors for RA. Our data are in line with the previous studies that level of IL-37 is correlated with RA activity. ${ }^{15,20,21}$ On top, our study correlates the levels of IL-37 with the severity of RA in a Chinese population.

Recently, growing evidence has implicated the role of IL37 as a natural suppressor in rheumatic autoimmune diseases. IL-37 is long considered as a cytokine that possesses dualfunctions in both intracellular and extracellular compartments. ${ }^{5}$ It has been shown to suppress inflammation in a number of disease models, including lipopolysaccharideinduced shock, ${ }^{9}$ hepatic ischemia/reperfusion (I/R) injury ${ }^{10}$ and obesity-induced inflammation and insulin resistance. ${ }^{7}$ Elevated level of IL-37 was observed in several autoimmune diseases, such as chronic hepatitis B virus infection, ${ }^{22}$ chronic inflammatory bowel disease, ${ }^{23}$ and systemic lupus erythematosus. ${ }^{24}$ At the meantime, decreased IL-37 expression was detected in patients with intervertebral disc degeneration. ${ }^{25}$ Multiple studies have suggested the correlation between IL-37 level and pro-inflammatory cytokine production. Immunohistochemical staining of the synovial lining of RA patients has shown elevated IL-37 level as compared to healthy controls. ${ }^{9}$ IL-37 and other pro-inflammatory cytokines are known to mutually affect each other in RA patients. ${ }^{21,26}$ Despite of the increasing levels of IL-37 in RA patients, they are still relatively low compared to other pro-inflammatory cytokines, which might be a possible reason that underlies RA progression. ${ }^{27}$ In addition, IL- 37 has been proven to inhibit the expression of pro-inflammatory cytokines such as IL-1, IL6 , IL- 8 , IL-17, IL-23, TNF- $\alpha$, and IFN- $\gamma{ }^{28}$ Therefore, it is reasonable to assume that the uncontrolled inflammation in RA is a result of inadequate anti-inflammation cytokines, such as IL-37. ${ }^{21}$ In line with this notion, decreased level of serum IL37 was observed in remission RA patients majorly regulated by the pro-inflammatory cytokines. ${ }^{13}$ Taking into account of our results and these previous studies, it is possible that during RA progression increased amount of IL-37 is produced to inhibit the elevated level of inflammation, likely via suppression of pro-inflammatory cytokines.

There are a few limitations in the current study. First, we could not investigate the serum and cellular levels of other inflammatory-related cytokines and their correlation with RA progression, as well as the IL-37 level. It is worth noting that correlation of other cytokines with RA progression will not affect our current conclusion on IL-37, since the mechanism of action of individual cytokines is relatively specific. Second, future study should focus on investigating the molecular mechanisms underlying the role of IL-37 in RA progression. Third, our current study only studied the Chinese RA population. Further studies on other ethnic groups and bigger patient populations are necessary. Fourth, due to the nature of this singleinstitution study, the sample size is rather limited. Future multi-institutional studies with larger sample size are needed to solidify our findings.

In summary, our study shows that serum IL-37 level and expression levels of IL-37 in PBMC and synovial cell are positively correlated with RA progression, where the former can be used as a potential biomarker for RA severity diagnosis when patients are admitted to the hospital for blood test.

\section{Data Sharing Statement}

All data generated or analyzed during this study are included in the manuscript.

\section{Consent for Publication}

All authors have read and approved the manuscript.

\section{Author Contributions}

All authors made a significant contribution to the work reported, whether that is in the conception, study design, execution, acquisition of data, analysis and interpretation, or in all these areas; took part in drafting, revising or critically reviewing the article; gave final approval of the version to be published; have agreed on the journal to which the article has been submitted; and agree to be accountable for all aspects of the work.

\section{Funding}

There is no funding to report.

\section{Disclosure}

The authors declare that they have no competing interests.

\section{References}

1. Smolen JS, Aletaha D, McInnes IB. Rheumatoid arthritis. Lancet. 2016;388(10055):2023-2038. doi:10.1016/S0140-6736(16)30173-8 
2. Brennan FM, McInnes IB. Evidence that cytokines play a role in rheumatoid arthritis. J Clin Invest. 2008;118(11):3537-3545. doi:10.1172/ JCI36389

3. Nam JL, Takase-Minegishi K, Ramiro S, et al. Efficacy of biological disease-modifying antirheumatic drugs: a systematic literature review informing the 2016 update of the EULAR recommendations for the management of rheumatoid arthritis. Ann Rheum Dis. 2017;76 (6):1113-1136. doi:10.1136/annrheumdis-2016-210713

4. Matsui T, Kuga Y, Kaneko A, et al. Disease Activity Score 28 (DAS28) using C-reactive protein underestimates disease activity and overestimates EULAR response criteria compared with DAS28 using erythrocyte sedimentation rate in a large observational cohort of rheumatoid arthritis patients in Japan. Ann Rheum Dis. 2007;66 (9):1221-1226. doi:10.1136/ard.2006.063834

5. Bulau AM, Nold MF, Li S, et al. Role of caspase-1 in nuclear translocation of IL-37, release of the cytokine, and IL-37 inhibition of innate immune responses. Proc Natl Acad Sci U S A. 2014;111 (7):2650-2655. doi:10.1073/pnas.1324140111

6. Jia HL, Liu J, Han B. Reviews of interleukin-37: functions, receptors, and roles in diseases. Biomed Res Int. 2018;2018. doi:10.1155/2018/ 4793971

7. Ballak DB, van Diepen JA, Moschen AR, et al. IL-37 protects against obesity-induced inflammation and insulin resistance. Nat Commun. 2014;5:4711. doi:10.1038/ncomms5711

8. McNamee EN, Masterson JC, Jedlicka P, et al. Interleukin 37 expression protects mice from colitis. Proc Natl Acad Sci U S A. 2011;108 (40):16711-16716. doi:10.1073/pnas.1111982108

9. Nold MF, Nold-Petry CA, Zepp JA, Palmer BE, Bufler P, Dinarello CA. IL-37 is a fundamental inhibitor of innate immunity. Nat Immunol. 2010;11(11):1014-U64. doi:10.1038/ni.1944

10. Sakai N, Van Sweringen HL, Belizaire RM, et al. Interleukin-37 reduces liver inflammatory injury via effects on hepatocytes and non-parenchymal cells. $J$ Gastroenterol Hepatol. 2012;27 (10):1609-1616. doi:10.1111/j.1440-1746.2012.07187.x

11. Teng X, Hu Z, Wei X, et al. IL-37 ameliorates the inflammatory process in psoriasis by suppressing proinflammatory cytokine production. J Immunol. 2014;192(4):1815-1823. doi:10.4049/jimmunol.1300047

12. Yang L, Zhang J, Tao J, Lu T. Elevated serum levels of interleukin-37 are associated with inflammatory cytokines and disease activity in rheumatoid arthritis. APMIS. 2015;123(12):1025-1031. doi:10.1111/apm.12467

13. Zhao PW, Jiang WG, Wang L, Jiang ZY, Shan YX, Jiang YF. Plasma levels of IL-37 and correlation with TNF-alpha, IL-17A, and disease activity during DMARD treatment of rheumatoid arthritis. PLoS One. 2014;9(5).

14. Dayer JM, Krane SM, Russell RG, Robinson DR. Production of collagenase and prostaglandins by isolated adherent rheumatoid synovial cells. Proc Natl Acad Sci U S A. 1976;73(3):945-949. doi:10.1073/pnas.73.3.945
15. Yuan ZC, Wang JM, Huang AF, Su LC, Li SJ, Xu WD. Elevated expression of interleukin-37 in patients with rheumatoid arthritis. Int $J$ Rheum Dis. 2019;22(6):1123-1129. doi:10.1111/1756-185X.13539

16. Ding L, Hong X, Sun B, et al. IL-37 is associated with osteoarthritis disease activity and suppresses proinflammatory cytokines production in synovial cells. Sci Rep. 2017;7(1):11601. doi:10.1038/s41598-01711397-5

17. Baecklund E, Iliadou A, Askling J, et al. Association of chronic inflammation, not its treatment, with increased lymphoma risk in rheumatoid arthritis. Arthritis Rheum. 2006;54(3):692-701. doi:10.1002/art.21675

18. Wolfe F. The natural history of rheumatoid arthritis. J Rheumatol Suppl. 1996;44:13-22.

19. Ye L, Jiang B, Deng J, et al. IL-37 alleviates rheumatoid arthritis by suppressing IL-17 and IL-17-triggering cytokine production and limiting Th17 cell proliferation. J Immunol. 2015;194(11):5110-5119. doi:10.4049/jimmunol.1401810

20. Ragaba D, Mobasher S, Shabaan E. Elevated levels of IL-37 correlate with $\mathrm{T}$ cell activation status in rheumatoid arthritis patients. Cytokine. 2019;113:305-310. doi:10.1016/j.cyto.2018.07.027

21. Xia T, Zheng XF, Qian BH, et al. Plasma interleukin-37 is elevated in patients with rheumatoid arthritis: its correlation with disease activity and Th1/Th2/Th17-related cytokines. Dis Markers. 2015;2015:1-6. doi: $10.1155 / 2015 / 795043$

22. Li C, Ji H, Cai Y, et al. Serum interleukin-37 concentrations and $\mathrm{HBeAg}$ seroconversion in chronic HBV patients during telbivudine treatment. J Interferon Cytokine Res. 2013;33(10):612-618. doi:10.1089/jir.2013.0001

23. Weidlich S, Bulau AM, Schwerd T, et al. Intestinal expression of the anti-inflammatory interleukin-1 Homologue IL-37 in pediatric inflammatory bowel disease. J Pediatr Gastr Nutr. 2014;59(2):E18E26. doi:10.1097/MPG.0000000000000387

24. Song LJ, Qiu F, Fan YC, et al. Glucocorticoid regulates interleukin-37 in systemic lupus erythematosus. J Clin Immunol. 2013;33(1):111-117. doi:10.1007/s10875-012-9791-Z

25. Wan ZY, Sun Z, Song F, et al. Downregulated interleukin 37 expression associated with aggravation of intervertebral disc degeneration. Int J Clin Exp Pathol. 2014;7(2):656-662.

26. Boraschi D, Lucchesi D, Hainzl S, et al. IL-37: a new anti-inflammatory cytokine of the IL-1 family. Eur Cytokine Netw. 2011;22(3):127-147. doi:10.1684/ecn.2011.0288

27. Isomaki P, Punnonen J. Pro- and anti-inflammatory cytokines in rheumatoid arthritis. Ann Med. 1997;29(6):499-507. doi:10.3109/ 07853899709007474

28. Pan Y, Wen X, Hao D, et al. The role of IL-37 in skin and connective tissue diseases. Biomed Pharmacother. 2020;122:109705. doi:10.1016/ j.biopha.2019.109705
International Journal of General Medicine

\section{Publish your work in this journal}

The International Journal of General Medicine is an international, peer-reviewed open-access journal that focuses on general and internal medicine, pathogenesis, epidemiology, diagnosis, monitoring and treatment protocols. The journal is characterized by the rapid reporting of reviews, original research and clinical studies across all disease areas. The manuscript management system is completely online and includes a very quick and fair peer-review system, which is all easy to use. Visit http://www.dovepress.com/ testimonials.php to read real quotes from published authors. 